\title{
A Generalized Finite Element Method for Hydrodynamic Modeling of Short-channel Devices
}

\author{
MIN SHEN ${ }^{\mathrm{a}}$, MING-C. CHENG ${ }^{\mathrm{b}, *}$ and J. J. LIOU \\ ${ }^{a}$ Advanced Materials Research Institute, University of New Orleans, New Orleans, LA 70148, USA; \\ ${ }^{\mathrm{b}}$ Department of Electrical and Computer Engineering, Box 5720, Clarkson University, \\ Potsdam, NY 13676, USA; \\ ${ }^{\mathrm{c}}$ Department of Electrical and Computer Engineering, University of Central Florida, \\ Orlando, FL 32816, USA
}

\begin{abstract}
A finite element method based on the least-squares scheme is developed for hydrodynamic simulation of two-dimensional short-channel semiconductor devices. Although this general-purpose finite element method has been shown in fluid dynamics to be more universal to flow problems than other finite element approaches and has been applied in recent years to a wide range of problems in fluid dynamics, it is still unfamiliar to the semiconductor device community. Application of the developed hydrodynamic least squares finite element method (LSFEM) to simulation of a 2D MESFET with a deepsubmicron gate has demonstrated its robustness and effectiveness for the hydrodynamic device simulation.
\end{abstract}

Keywords: Hydrodynamic model; Device simulation; Least squares finite element

\section{INTRODUCTION}

Because of the similarity between the semiconductor hydrodynamic equations and NavierStokes/Euler equations, some numerical methods that have been successfully applied to hydrodynamic simulation of semiconductor devices are derived from the fluid dynamics. Although the least squares finite element method (LSFEM) is one of the effective numerical schemes that have been used in a wide range of fluid dynamics problems [1-5], it is still new to the semiconductor device community. The least-squares finite element formulation possesses many advantages. The algebraic system derived in the LSFEM is symmetric and positive definite [1,2]. It allows adoption of equal-order interpolations for all independent variables, which makes the method simple to formulate and easy to program. The LSFEM is effective for handling convective problems because the method contains an upwind mechanism to stabilize the numerical solution of

*Corresponding author. Tel.: 1-315-268-7735, Fax: 1-315-268-7600, e-mail: mcheng@clarkson.edu 
the hyperbolic equations. Unlike the mixed Galerkin schemes, the LSFEM contains no adjustable parameter. Transport problems in the LSFEM can be handled in a very simple framework. Modification of the physical model in LSFEM amounts to simple subroutine changes in the computer code associated with the coefficient matrices and vectors.

In this study, the least squares finite element formulation is applied to the semiconductor hydrodynamic equations coupled with Poisson's equation, and the developed Hydrodynamic LSFEM is examined on a depletion-mode $n$-channel MESFET structure. The hydrodynamic system for simulation of semiconductor devices is presented in Section II, and formulation of the hydrodynamic LSFEM is given in Section III. In Section IV, numerical results and discussion are presented. Conclusion is drawn in Section V.

\section{HYDRODYNAMIC SYSTEM FOR SEMICONDUCTOR DEVICES}

In this study, the semiconductor hydrodynamic equations, including conservation equations of carrier density $n$, momentum $\mathbf{p}$ and energy $w$, are given as

$$
\begin{gathered}
\frac{\partial n}{\partial t}+\nabla \cdot(n \mathbf{v})=0 \\
\frac{\partial n \mathbf{p}}{\partial t}+\nabla \cdot n \mathbf{p v}+\nabla n k_{B} T_{e}=-e n \mathbf{E}-\frac{n \mathbf{p}}{\tau_{m}} \\
\frac{\partial n w}{\partial t}+\nabla \cdot n w \mathbf{v}+\nabla \cdot n k_{B} T_{e} \mathbf{v}+\nabla \cdot \mathbf{Q} \\
=-e n \mathbf{E} \cdot \mathbf{v}-\frac{n\left(w-w_{0}\right)}{\tau_{w}},
\end{gathered}
$$

where $\mathbf{v}$ is the electron velocity, $\mathbf{p}=m^{*} \mathbf{v}\left(m^{*}\right.$ is the electron mass), $\mathbf{E}$ is the electric filed, $T_{\mathrm{e}}$ is electron temperature, $k_{B}$ is the Boltzmann constant and $e$ is the elementary charge. $\mathbf{Q}$ is the heat flux described by the thermal conductivity $\kappa$ and temperature gradient:

$$
\mathbf{Q}=-\kappa \nabla T_{e} \quad \text { and } \quad \kappa=\frac{5 k_{B}^{2} T_{0} \tau_{m}}{2 m^{*}}
$$

The carrier energy $w$ is related to the carrier temperature and velocity by

$$
w=\frac{3}{2} k_{B} T_{e}+\frac{1}{2} m^{*} \mathbf{v}^{2}
$$

The thermal energy $w_{o}$ is calculated from $w_{o}=$ $1.5 \mathrm{k}_{\mathrm{B}} T_{o}$, where $T_{o}$ is the lattice temperature. The electric field $\mathbf{E}$ is obtained from the gradient of electric potential $\psi$

$$
\mathbf{E}=-\nabla \psi
$$

and the potential is determined from the Poisson's equation

$$
\nabla^{2} \psi=-\frac{\rho}{\varepsilon_{s}}
$$

where $\varepsilon_{s}$ is the dielectric constant of the semiconductor, and $\rho$ is the charge density in the semiconductor device. Simulation of an $n$-channel MESFET in this paper is based on the one carrier hydrodynamic model, and thus $\rho=q\left(N_{d}-n\right)$, where $N_{d}$ is the $n$-type impurity density.

In this model, the coefficients, $\tau_{m}, \tau_{w}$, and $m^{*}$, in the hydrodynamic equations are assumed to be energy dependent. Their values are extracted from the Monte Carlo simulation. Equations (1) $-(7)$, together with these energy-dependent coefficients, form a closed set of hydrodynamic system.

\section{LEAST-SQUARE FINITE ELEMENT FORMULATION}

The hydrodynamic system described by Eqs. (1) -(7) can be solved if $n, \mathbf{v}, w$ and $\psi$ are chosen to be the independent variables. This however will lead to second-order differentiation for $w$ and $\psi$. To be able to use linear shape functions [1,2] for the elements of all variables 
in the LSFEM, additional independent variables $\mathbf{Q}$ and $\mathbf{E}$ are selected in the hydrodynamic system. Based on this approach, the hydrodynamic model described by Eqs. (1)-(7) can be expressed by a unified matrix equation

$$
[\mathbf{G}] \frac{\partial \mathbf{U}}{\partial t}+\left[\mathbf{A}_{x}\right] \frac{\partial \mathbf{U}}{\partial x}+\left[\mathbf{A}_{y}\right] \frac{\partial \mathbf{U}}{\partial y}+\left[\mathbf{A}_{o}\right] \mathbf{U}=\mathbf{H}
$$

where $\mathbf{U}$ is the state variable vector and defined, for a $2 \mathrm{D}$ system, as

$$
\mathbf{U}=\left[\begin{array}{lllllllll}
n & v_{x} & v_{y} & w & \psi & E_{x} & E_{y} & Q_{x} & Q_{y}
\end{array}\right]^{T} .
$$

Using backward difference with linearization of the coefficient matrices $\left[\mathbf{A}_{x}\right]$ and $\left[\mathbf{A}_{y}\right]$ at a given time step $\Delta t=t^{k+1}-t^{k}$, Eq. (8) can be approximated by

$$
\begin{aligned}
{[\mathbf{G}] \mathbf{U}^{k+1} } & -[\mathbf{G}] \mathbf{U}^{k}+\Delta t\left[\mathbf{A}_{x}\right]^{k} \frac{\partial \mathbf{U}^{k+1}}{\partial x} \\
& +\Delta t\left[\mathbf{A}_{y}\right]^{k} \frac{\partial \mathbf{U}^{k+1}}{\partial y} \\
& +\Delta t\left[\mathbf{A}_{o}\right]^{k} \mathbf{U}^{k+1}=\Delta t \mathbf{H}^{k}
\end{aligned}
$$

Based on Eq. (8), $\mathbf{U}^{k+1}$ is therefore determined from the vector $\mathbf{U}^{k}$ at the previous time step. The residual of the difference equation in Eq. (10) is defined as:

$$
\begin{aligned}
\Re= & {[\mathbf{G}] \mathbf{U}^{k+1}-[\mathbf{G}] \mathbf{U}^{k}+\Delta t\left[\mathbf{A}_{x}\right]^{k} \frac{\partial \mathbf{U}^{k+1}}{\partial x} } \\
& +\Delta t\left[\mathbf{A}_{y}\right]^{k} \frac{\partial \mathbf{U}^{k+1}}{\partial y} \\
& +\Delta t\left[\mathbf{A}_{o}\right]^{k} \mathbf{U}^{k+1}-\Delta t \mathbf{H}^{k} .
\end{aligned}
$$

If the exact solution is obtained, the residual will be zero. A functional is introduced to minimize the deviation between the numerical and exact solutions in the $2 \mathrm{D}$ domain $\Omega$,

$$
\xi=\int_{\Omega} \Re^{T} \Re d \Omega .
$$

When the optimum solution is obtained, the variation of the functional should be zero:

$$
\delta \xi=2 \int_{\Omega} \delta \Re^{T} \Re d \Omega=0 .
$$

The finite element global matrix and nodal vectors can be obtained from Eq. (13) [1,2]. Consequently, the whole problem reduces to solving the algebraic equations represented by a matrix equation $[1,2]$.

\section{NUMERICAL SIMULATION AND DISCUSSION}

The developed hydrodynamic LSFEM is applied to the simulation of a depletion-mode Si $n$-channel

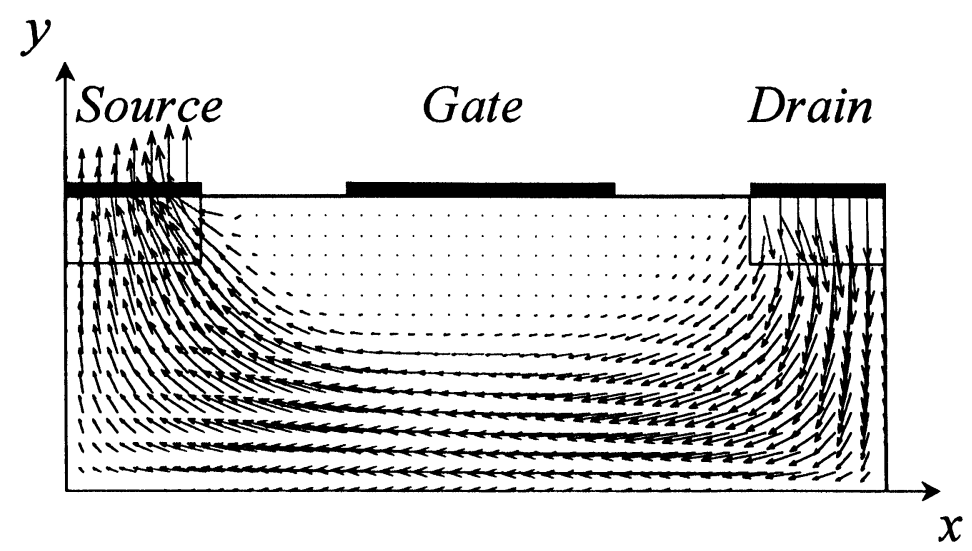

FIGURE 1 The 2D MESFET structure used in the hydrodynamic simulation. The thick lines denote the metal contacts. $N_{d}=$ $10^{17} \mathrm{~cm}^{-3}$ in the device except for the $n^{+}$source/drain region where $N_{d}=3 \times 10^{17} \mathrm{~cm}^{-3}$. The current density vectors are also included at $V_{d s}=2 \mathrm{~V}$ and $V_{g s}=-0.6 \mathrm{~V}$. 
MESFET with a gate length of $0.2 \mu \mathrm{m}$ to examine its stability and effectiveness. The device structure is shown in Figure 1, where the gate is located at the center between source and drain contacts, and the drain/source metal contact is $0.1 \mu \mathrm{m}$ long. The device length and depth are 0.6 and $0.2 \mu \mathrm{m}$, respectively. The depth of the $n^{+}$source/drain region is $0.05 \mu \mathrm{m}$. The hydrodynamic simulation of the MESFET is carried out at $V_{d s}=2.0 \mathrm{~V}$ and $V_{g s}=-0.6 \mathrm{~V}$. Current density vectors in the MESFET are also included in Figure 1 where the current flow in the channel is clearly displayed, and the depletion region due to the Schottky barrier is observed. The potential profile in the device is shown in Figure 2. A steep Schottky barrier at a reverse-biased gate voltage is illustrated.

Due to the reverse-biased Schottky barrier, Figure 3 shows that the electron concentration near $y=0.2 \mu \mathrm{m}$ decreases approximately by 10 orders of magnitude from the source/drain contact to the gate depletion region within a very short distance. An equilibrium boundary condition is assumed for the electron concentration; that is the gate current is neglected in

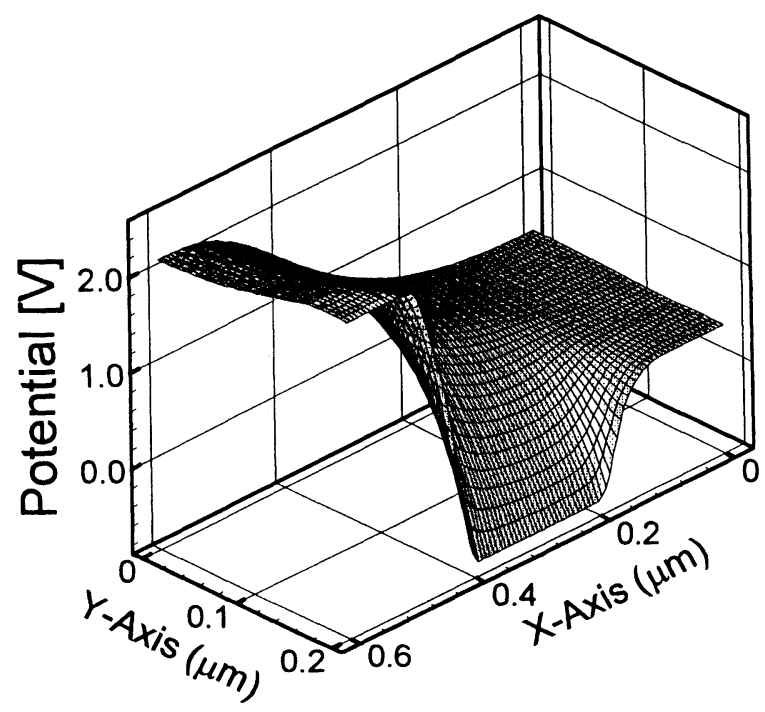

FIGURE 2 Potential in the MESFET at $V_{d s}=2.0 \mathrm{~V}$ and $V_{g s}=-0.6$. the simulation. Lattice temperature $(300 \mathrm{~K})$ is assumed for electron temperature at all metal contacts, and as can be seen in Figure 4 this leads to sudden changes in electron temperature at the metal edges, particularly near $x=0.4 \mu \mathrm{m}$. The large electron temperature between metal contacts along $y=0.2 \mu \mathrm{m}$ is caused by an extremely large electric field, as shown in Figure 5(a). In addition,

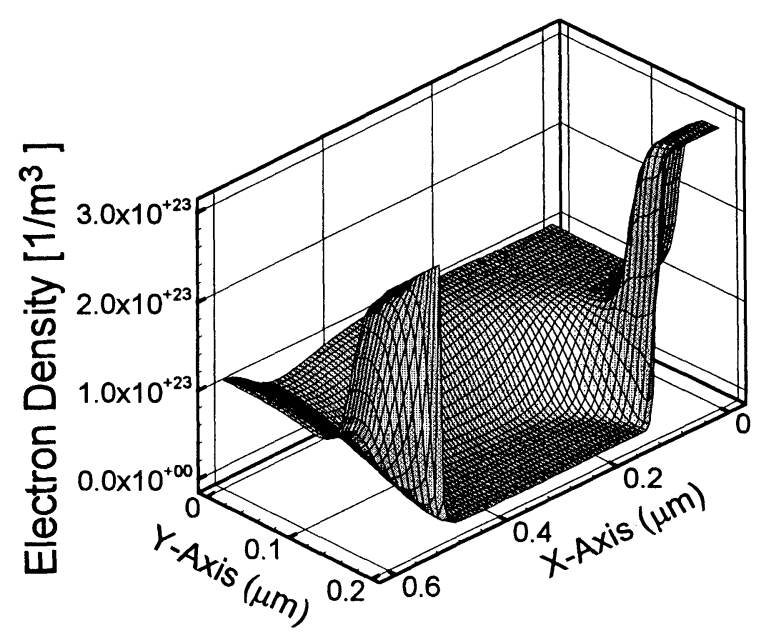

FIGURE 3 Electron density in the MESFET at $V_{d s}=2.0 \mathrm{~V}$ and $V_{g s}=-0.6$.

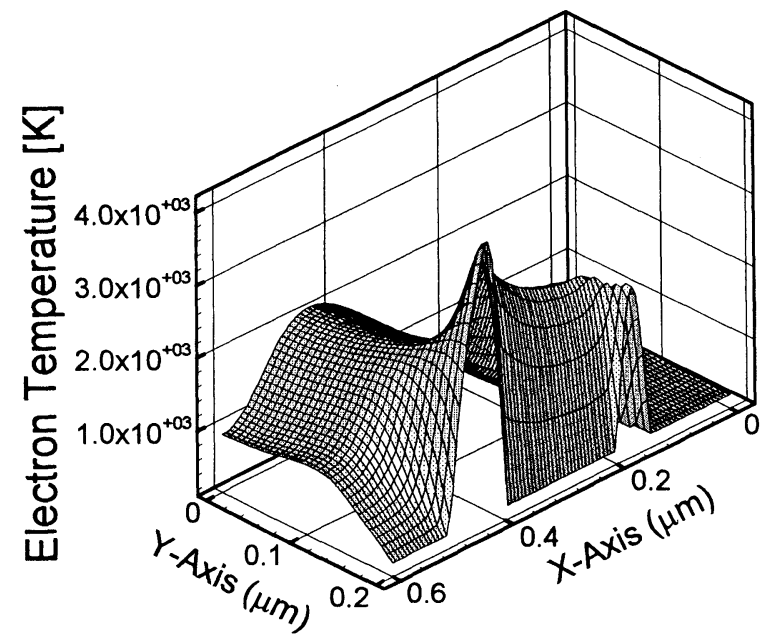

FIGURE 4 Electron temperature in the MESFET at $V_{d s}=$ $2.0 \mathrm{~V}$ and $V_{g s}=-0.6$. 

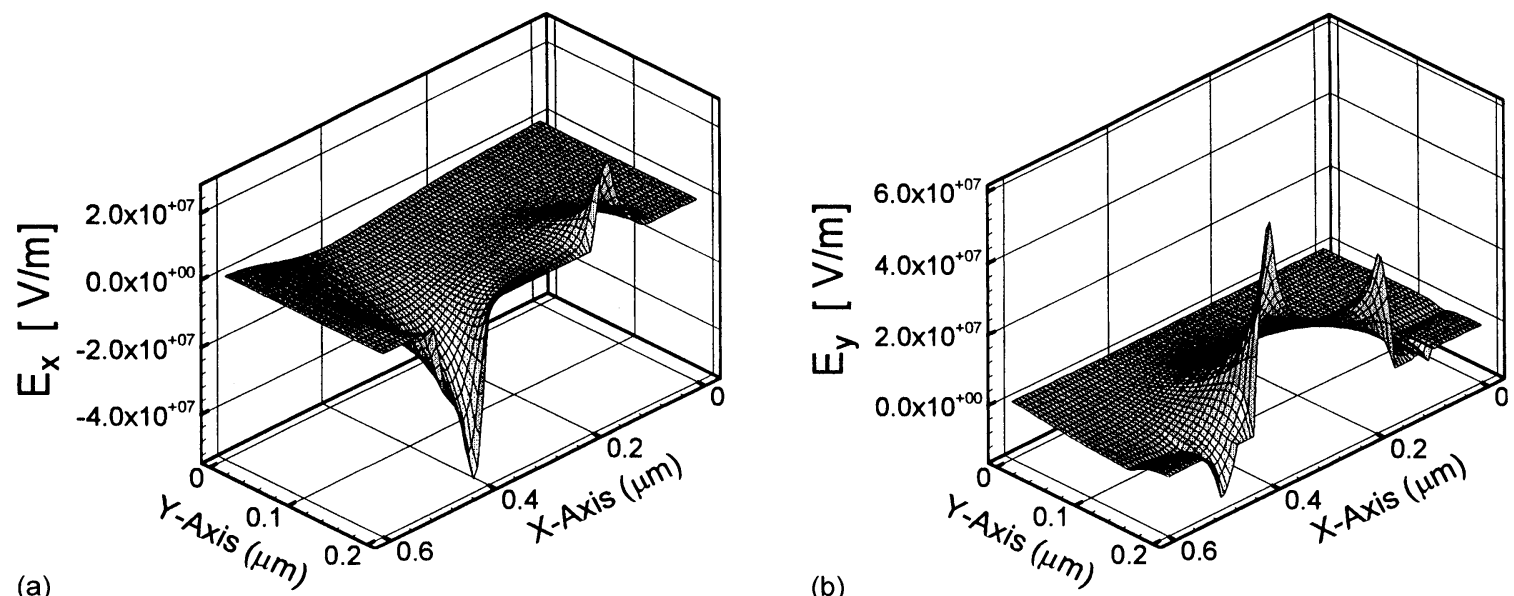

FIGURE 5 (a) $x$-component and (b) $y$-component electric fields in the MESFET at $V_{d s}=2.0 \mathrm{~V}$ and $V_{g s}=-0.6$.

because of the lattice temperature assumption for electrons and the large transverse electric field under the gate resulting from the steep Schottky barrier, electron temperature drastically increases from the gate toward the channel. Although the maximum electron temperature in the channel is only near $2000 \mathrm{~K}$, an electron temperature as high as $4000 \mathrm{~K}$ is found near the gate.

These numerical results illustrate that the upwind mechanism in the LSFEM is able to stabilize the convective flux in the semiconductor hydrodynamic equations. Moreover, the LSFEM has shown to be effective at handling large discontinuities in the electron concentration, mean energy and electric fields near the metal boundaries, as shown in Figures 1-4.

\section{CONCLUSION}

The LSFEM [1,2] has been applied to a hydrodynamic system including electron density, energy, and momentum conservation equations coupled with Poisson's equation. A 2D MESFET structure given in Figure 1 has been used to examine the stability and effectiveness of the developed hydrodynamic LSFEM. Unlike other finite element schemes developed for hydrodynamic modeling of semiconductor devices [6-8], no adjustable parameter or smooth operator is involved in the developed LSFEM to arrive at stable solution. In summary, the LSFEM has been shown to be simple, stable and robust for hydrodynamic simulation of $2 \mathrm{D}$ semiconductor devices.

\section{Acknowledgement}

The authors Cheng and Liou are partially supported by SRC under Grant Number 2000RJ-873G.

\section{References}

[1] Bo-Nan Jiang and Louis A. Povinelli (1990). "LeastSquares Finite Element Method for Fluid Dynamics", Comput. Methods Appl. Mech. Engrg., 81, 13.

[2] Bo-Nan Jiang and Chang, C. L. (1990). "Least-Squares Finite Elements for the Stokes Problem", Comput. Methods Appl. Mech. Engrg., 78, 297.

[3] Thomas A. Manteuffel and Klaus J. Ressel (1998). "LeastSquares Finite-Element Solution of the Neutron Transport Equation in Diffusive Regimes", SIAM J. Numer. Analysis, 35, 806.

[4] Pavel B. Bochev (1997). "Analysis of Least-Squares Finite Element Methods for the Navier-Stokes Equations", SIAM J. Numer. Analysis, 34, 1817. 
[5] Nguyen, H. and Reynen, J. (1984). "A space-time leastsquares finite element scheme for advection-diffusion equations", Comput. Meth. Appl. Mech. Engrg., 42, 331.

[6] Fortin, M. and Yang, G. (1996). "Simulation of the hydrodynamic model of semiconductor devices by a finite element method", Compel, 15, 4.
[7] Chen, Z. (1996). A finite element method for the quantum hydrodynamic model for semiconductor devices, Compters Math. Applic., 31, 17.

[8] Aluru, N. R., Raefsky, A., Pinsky, P. M. et al. (1993). “A finite element formulation for the hydrodynamic semiconductor device equations", Computer Methods in Appl. Mech. and Engrg., 107, 269. 

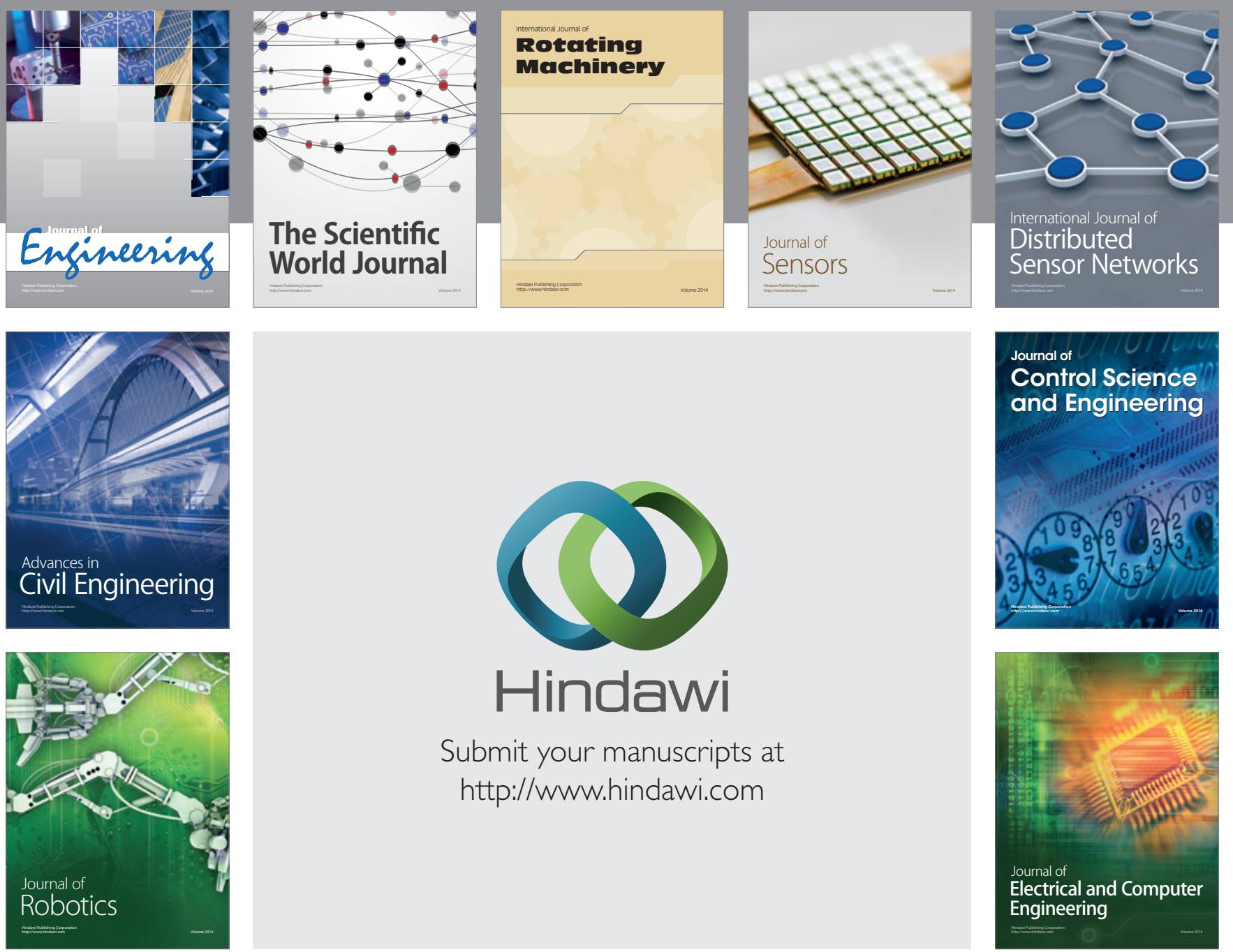

Submit your manuscripts at

http://www.hindawi.com
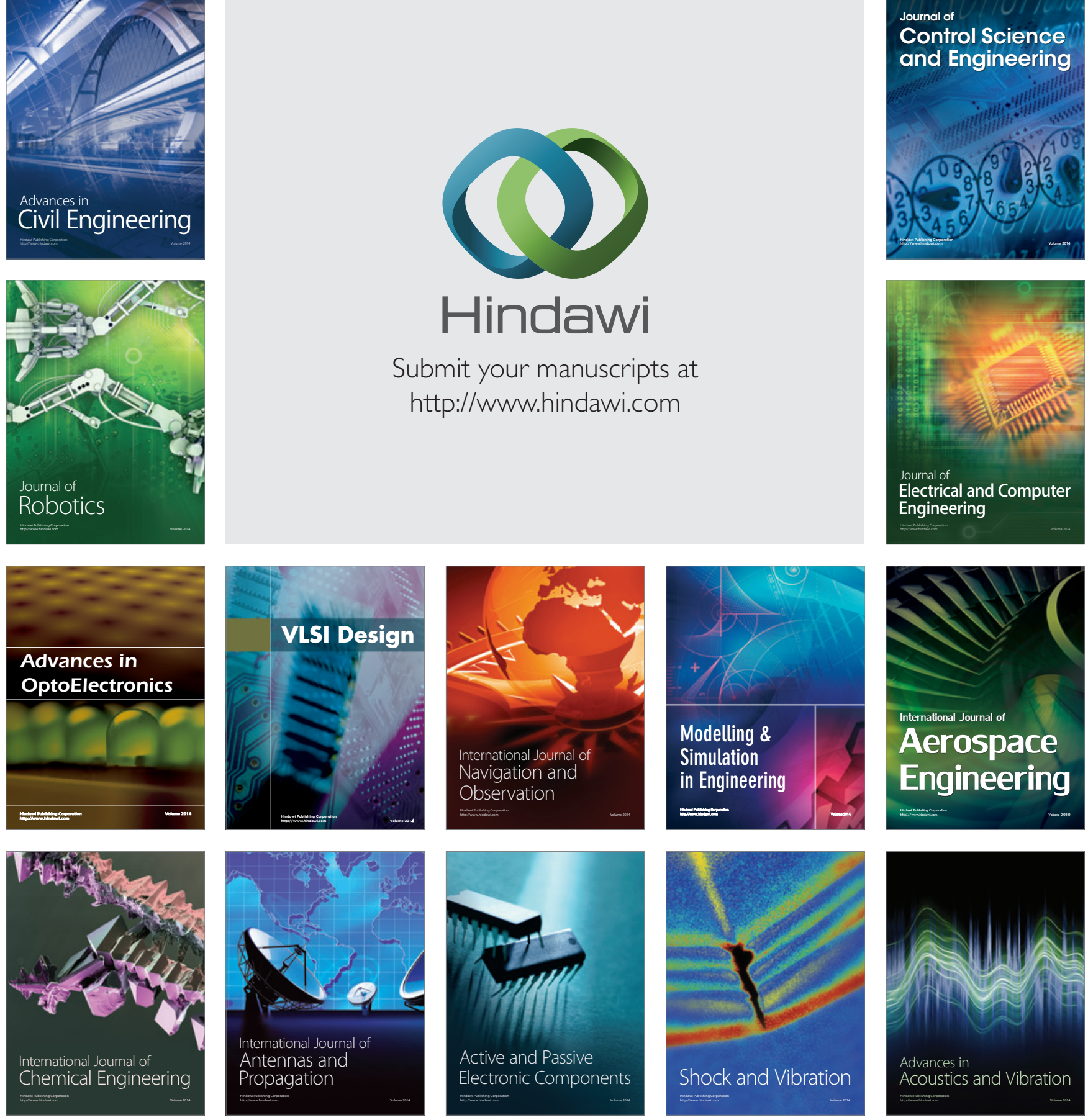